\title{
The relationship between acid and bile reflux and symptoms in gastro-oesophageal reflux disease
}

\author{
R E K Marshall, A Anggiansah, W A Owen, W J Owen
}

\begin{abstract}
Background-The role of bile in the genesis of oesophageal symptoms and disease is incompletely understood. A new method of ambulatory bile monitoring may help to define this role.

Aims-To establish the relationship between symptom events and acid and bile reflux episodes.

Patients-59 consecutive patients presenting for further investigation of gastrooesophageal reflux disease.

Methods-All patients underwent combined ambulatory pH and bile monitoring. For each patient, a symptom index (SI) was calculated in relation to both acid reflux and bile reflux episodes.

Results-Patients were divided into those without (group 1, $n=21$ ) and those with (group 2, $n=38$ ) acid reflux. A total of 394 symptoms were identified in 59 patients. In group 1 , there were fewer symptom events per patient (mean $4 \cdot 1$ ) than group 2 (mean 8:1). Twenty three per cent of symptom events were associated with acid reflux in group 1 and $41 \%$ in group 2 . Only $6 \%$ of symptom events in both groups were related to bile reflux. In group 1 both the acid and bile related SI score were low. In group 2 the bile related SI score was low, but the acid related SI score was high.

Conclusions-Symptoms are much more often related to acid reflux than bile reflux. Bile reflux does not seem to be a major factor in producing oesophageal symtoms.
\end{abstract}

(Gut 1997; 40: 182-187)

Keywords: gastro-oesophageal reflux, symptom index, bile monitoring.

While there is no doubt that gastrooesophageal reflux of acid is responsible for the production of oesophagitis and its complications, the role of duodenal contents (specifically trypsin and bile acids) in oesophageal mucosal disease remains less certain. Both animal and human studies have suggested a role for bile in the pathogenesis of oesophagitis, ${ }^{12}$ Barrett's oesophagus, ${ }^{3}{ }^{4}$ and oesophageal adenocarcinoma, ${ }^{5}$ but the difficulty so far has been in trying to quantify bile reflux in vivo. In the past various methods have been used but they all have their drawbacks. HIDA scintigraphy has been used, but this is a comparatively insensitive and unphysiological method for the measurement of gastrooesophageal reflux. ${ }^{6}$ Aspiration studies have also been performed to determine the quantity of bile acids in oesphageal refluxate, but this is cumbersome, invasive, and as with scintigraphy it is unphysiological, measuring short time periods in static conditions. Ambulatory 24 hour $\mathrm{pH}$ monitoring has been used, the assumption being that an alkaline shift in $\mathrm{pH}$ $>7$ equates with the presence of duodenal contents. However, combined aspiration and $\mathrm{pH}$ monitoring studies have shown this not to be the case. ${ }^{7}$

Bilitec 2000, a fibreoptic spectrophotometric probe, was developed to overcome these problems. ${ }^{8}$ Bilirubin has a characteristic absorbance spectrum at $453 \mathrm{~nm}$, and by continuous monitoring of the oesophageal refluxate, the presence of bilirubin in the refluxate can be determined on an ambulatory basis. In this way Bilitec 2000 uses bilirubin as a marker for the presence of duodenal contents. Extensively validated both in vitro and in vivo, ${ }^{9-11}$ it has been shown that gastrooesophageal bile reflux can be monitored in a relatively patient friendly manner, and that in patients with normal gastro-intestinal anatomy, acid and bile reflux times correlate extremely well, and that there is no relationship between bile and alkaline reflux. ${ }^{12-14}$

The aim of the study was to analyse the symptom events in a group of patients with gastro-oesophageal reflux disease (GORD), with regards to whether they were associated with episodes of acid or bile reflux. The simplest form of symptom analysis, the symptom index (SI), was used, to identify whether bile reflux is as potent a cause of symptoms as acid reflux.

\section{Methods}

\section{Patients}

Fifty nine consecutive patients (36 female; mean age 46.6 years; range 23 to 76 ) referred to the Oesophageal Laboratory at Guy's Hospital for further investigation of GORD were included in this study. All patients were complaining of heartburn or acid regurgitation, or both. None had previously undergone gastric or oesophageal surgery. Seven patients had undergone previous open cholecystectomy. All patients had stopped acid suppression therapy (either 48 hours beforehand for those taking $\mathrm{H}_{2}$ receptor antagonists, or seven days beforehand for those using proton pump inhibitors).

\section{Study design}

Static manometry was carried out beforehand in all cases. The nasal mucosa was sprayed with lignocaine, and a solid state pressure 
manometer (Gaeltec Ltd, Isle of Skye, UK) with six transducers, at $5 \mathrm{~cm}$ intervals and radially oriented at $90^{\circ}$, was introduced. Oesophageal manometry was then carried out using the station pull through technique to determine lower oesophageal sphincter (LOS) position and pressure, and to exclude patients with primary oesophageal motility disorders. A Berstein acid perfusion test was carried out.

After manometry, a dual channel antimony pH electrode $(2 \cdot 1 \mathrm{~mm}$ diameter $)$ and a fibreoptic bile probe $(3 \mathrm{~mm}$ diameter) (both Synectics Medical, Stockholm, Sweden) were strapped together using $12.5 \mathrm{~mm}$ Micropore tape (3M Medical-Surgical Division, St Paul, $M N)$, taking care not to obstruct the gap in the bile probe tip or the $\mathrm{pH}$ sensors. Before and after each study, the $\mathrm{pH}$ probes were calibrated in buffer solutions of $\mathrm{pH} 7$ and $\mathrm{pH} 1$, and the bile probe calibrated in water. The proximal $\mathrm{pH}$ sensor was strapped next to the bile probe and these placed $5 \mathrm{~cm}$ above the upper border of the manometrically determined LOS, with the distal $\mathrm{pH}$ sensor $15 \mathrm{~cm}$ distal to this, in the gastric body. We have found a dual channel $\mathrm{pH}$ probe with the bile probe next to the proximal $\mathrm{pH}$ sensor much easier to insert than a single channel $\mathrm{pH}$ probe, which presents quite a large end when combined with a bile probe.

Both probes were attached to data collecting devices worn on a belt on the patients' waist. Patients were given a diary sheet to record the time and content of their meals, time of going to bed and getting up, and their symptoms. They were also asked to press a marker button to record their symptom events. Patients were asked to avoid acidic foods that may interfere with $\mathrm{pH}$ monitoring and dark coloured foods that interfere with bilirubin absorbance (a list was provided), and were consequently told the foodstuffs they could eat. They were encouraged to carry out their normal daily activities, and to return the following day for the probes to be removed. Both bile and acid reflux data were then downloaded onto a computer for analysis.

The 59 patients were divided into two groups: group 1, those without significant acid reflux ( $\mathrm{n}=21(36 \%), 17$ female), and group 2, those with significant acid reflux $(n=38(64 \%)$, 19 male (Table I)).

\section{Data analyis}

Data were analysed on commercially available software (EsopHogram, Gastrosoft Inc, USA). Analysis of the 24 hour tracing was divided into total, upright interprandial, meal, postprandial, and supine periods. Acid reflux was

TABLE I Acid and bile reflux data for group 1 (no significant acid reflux) and group 2 (significant acid reflux)

\begin{tabular}{llc}
\hline & $\begin{array}{l}\text { Acid reflux } \\
(\% \text { of total time } \\
p H<4)\end{array}$ & $\begin{array}{l}\text { Bile reflux } \\
(\% \text { of total time } \\
\text { absorbance }>0 \cdot 14)\end{array}$ \\
\hline $\begin{array}{l}\text { Group 1 (n=21) } \\
\begin{array}{l}\text { No significant acid reflux) } \\
\text { Group 2 }(\mathrm{n}=38)\end{array}\end{array}$ & $0 \cdot 8(0 \cdot 2-1 \cdot 4)$ & $0 \cdot 1(0-1 \cdot 7)$ \\
(Significant acid reflux) & $7 \cdot 7(4 \cdot 7-15 \cdot 4)$ & $13 \cdot 25(5 \cdot 3-26 \cdot 5)$ \\
\hline
\end{tabular}

Values expressed as median (interquartile range). defined as a drop in intraoesophageal $\mathrm{pH}<4$, and bile reflux as bilirubin absorbance $>0 \cdot 14$. Significant gastro-oesophageal reflux was said to have occurred if the DeMeester score was greater than $14 \cdot 7$. Each symptom event was analysed: acid reflux was said to be associated with the symptom event if the $\mathrm{pH}$ dropped below 4 for more than 10 seconds in the two minutes either side of the symptom event, and bile reflux was said to have occurred if the bilirubin absorbance was greater than $0 \cdot 14$ for more than 10 seconds in the two minutes either side of the symptom event.

For each patient the SI was calculated for bile reflux:

SI $=\frac{\text { number } \text { of symptoms with bilirubin absorbance }>0 \cdot 14}{\text { total number of symptoms }} \times 100$

and the SI for acid reflux was calculated:

$$
\mathrm{SI}=\frac{\text { number } \text { of symptoms with } \mathrm{pH}<4}{\text { total number of symptoms }} \times 100
$$

This percentage was then converted to a SI score in the following manner: $0 \%=0$, $1-24 \%=1,25-49 \%=2,50-74 \%=3,75-99 \%=4$, $100 \%=5$.

\section{Statistical analysis}

Values are expressed as mean (SD) for parametric data and median (interquartile range) for non-parametric data. The Mann-Whitney $\mathrm{U}$ test was used to establish differences between two sets of non-parametric data, Student's $t$ test for parametric data, and correlations were established using the Spearman rank test. A p value $<0.05$ was considered significant.

\section{Results}

$p H$ and bile reflux data

For the group 1 patients, the median (interquartile range) total time $\mathrm{pH}<4$ was $0.8 \%$ $(0 \cdot 2-1 \cdot 4 \%)$ and bilirubin absorbance $>0 \cdot 14$ was $0 \cdot 1 \%(0 \%-1 \cdot 7 \%)$. The median (interquartile range) DeMeester score was 3.8 $(1 \cdot 4-5 \cdot 3)$. For the group 2 patients, the median (interquartile range) total time $\mathrm{pH}<4$ was $7 \cdot 7 \%(4 \cdot 7 \%-15 \cdot 4 \%)$ and bilirubin absorbance $>0.14$ was $13.25 \% \quad(5 \cdot 3 \%-26 \cdot 5 \%)$. The median (interquartile range) DeMeester score was $33.5(18 \cdot 7-61 \cdot 2)$. The correlation between total time $\mathrm{pH}<4$ and total time bilirubin absorbance $>0.14$ for all patients was extremely good: $r=0.55, \mathrm{p}<0.001$.

\section{Symptom and manometry data}

The various symptoms the patients complained of are listed in Table II. It can be seen that all patients complained of heartburn, and that belching and regurgitation were the next most frequent symptoms. Epigastric pain, dysphagia, and chest pain were less frequently complained of. However, the distribution of the symptoms between the two groups was comparable, the only difference being the greater frequency of chest pain in group 2 (those with significant acid reflux). 
TABLE II Occurrence of different symptoms among patients in group 1 and group 2

\begin{tabular}{lll}
\hline Symptom & Group 1 & Group 2 \\
\hline Heartburn & $21(100)$ & $38(100)$ \\
Belching & $15(71)$ & $27(71)$ \\
Rergurgitation & $11(52)$ & $23(60)$ \\
Dysphagia & $7(33)$ & $15(39)$ \\
Epigastric pain & $7(33)$ & $11(29)$ \\
Chest pain & $1(5)$ & $9(24)$ \\
\hline
\end{tabular}

Figures in parentheses are percentages

There was no difference in the frequency of a positive Bernstein test between the two groups, with 14 patients $(66 \%)$ in group 1 and 26 patients $(68 \%)$ in group 2 complaining of heartburn during intraoesophageal acid perfusion. There was a significant difference in the lower oesophageal sphincter pressure (LOSP) between the groups, the mean (SD) LOSP being $9 \cdot 19(4 \cdot 7) \mathrm{mm} \mathrm{Hg}$ in group 1 and 5.54 $(3.35) \mathrm{mm} \mathrm{Hg}$ in group $2(\mathrm{p}<0.001$, Student's $t$ test).

\section{Symptom analysis}

A total of 394 symptom events were identified in 59 patients (range 1-24 per patient) (Table III) Each event was defined by the time period in which it occurred (meal, postprandial, interprandial upright or supine) and by whether it was related to an episode of acid reflux, bile reflux, both acid and bile reflux, or neither. The majority of symptom events occurred in the upright interprandial (173 events, 44\%) and the postprandial (165 events, 42\%) periods, with 42 events $(11 \%)$ occurring in the supine period and only 14 events (3\%) occurring in the meal period.

With regards to the type of reflux associated with the symptom event, the greatest number of events was associated with neither acid nor bile reflux (178 events, $45 \%$ ). A total of 147 events $(37 \%)$ were associated with acid reflux alone, 45 events $(11 \%)$ were associated with both acid and bile reflux, and only 24 events $(7 \%)$ were associated with bile reflux alone.

In group 1,86 symptom events $(22 \%)$ occurred in 21 patients (36\%), an average of $4 \cdot 1$ symptom events per patient. In group 2 , 308 symptom events $(78 \%)$ occurred in 38 patients $(64 \%)$, an average of 8.1 symptom events per patient. Hence, symptoms were twice as frequent in those with significant acid reflux. When considering group 1 and group 2 separately, in each case the symptom events were distributed in a similar fashion between the time periods (the majority occurring in the postprandial and upright interprandial periods). However, there was a difference in

TABLE III Relation between the type of refluxate and symptom events, and the time period in which symptom events occurred

\begin{tabular}{lccccc}
\hline & $\begin{array}{l}\text { Acid } \\
\text { reflux }\end{array}$ & $\begin{array}{l}\text { Bile } \\
\text { reflux }\end{array}$ & $\begin{array}{l}\text { Both acid } \\
\text { and bile }\end{array}$ & Neither & \\
\hline Postprandial & 67 & 11 & 15 & 72 & $165(42)$ \\
Meal & 0 & 3 & 2 & 9 & $14(4)$ \\
Upright interprandial & 65 & 8 & 17 & 83 & $173(44)$ \\
Supine & 15 & 2 & 11 & 14 & $42(10)$ \\
& $147(37)$ & $24(60)$ & $45(11)$ & $178(45)$ & 394 \\
\hline
\end{tabular}

Figures in parentheses are percentages.

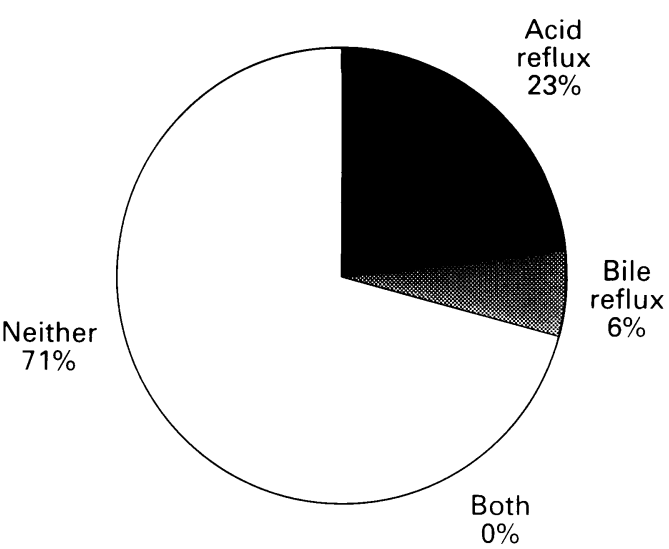

Figure 1: Nature of the refluxate associated with symptom events in patients without significant gastro-oesophageal acid reflux (group 1).

the type of refluxate associated with the symptom event: in group 1 (those without significant acid reflux), the majority of symptom events were associated with neither acid nor bile reflux (61 events, $71 \%$ ), with 20 events $(23 \%)$ associated with acid reflux and only five events (6\%) associated with bile reflux (Fig 1). In group 2 however (those with significant acid reflux), 127 symptom events ( $41 \%$ ) were associated with acid reflux, 45 symptom events $(15 \%)$ with both acid and bile reflux, only 19 symptom events $(6 \%)$ were associated with bile reflux alone and 117 symptom events $(38 \%)$ with neither acid nor bile reflux (Fig 2). Hence in group 2 there was a much higher proportion of symptoms associated with acid reflux than group 1, and in group 1 a much larger proportion were associated with neither acid nor bile reflux than in group 2, although in both groups there was a similarly small proportion of symptoms associated with bile reflux alone.

\section{Symptom index}

The symptom index was calculated for each patient, for both bile reflux and acid reflux. In group 1 (Fig 3), with regards to acid reflux, the SI score was 0 for 13 patients, 2 for five patients, 3 for two patients, and only one patient had an SI of 5. A similar picture is seen for bile reflux,

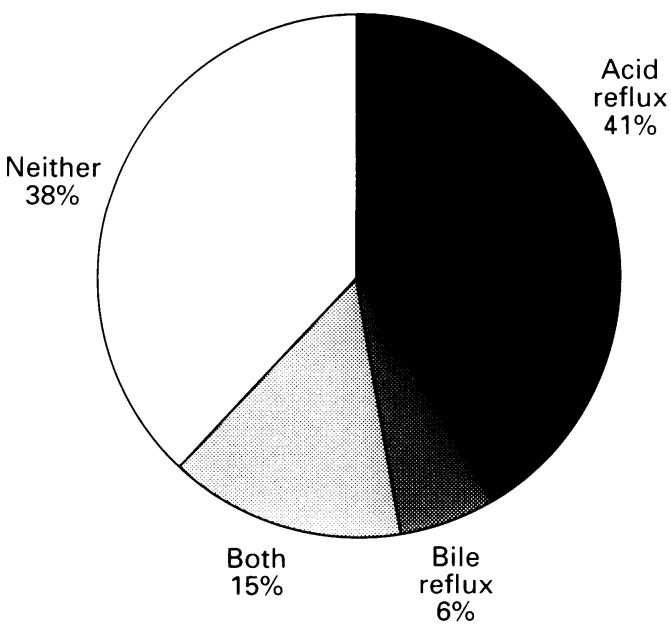

Figure 2: Nature of the refluxate associated with symptom events in patients with significant gastro-oesophageal acid reflux (group 2). 


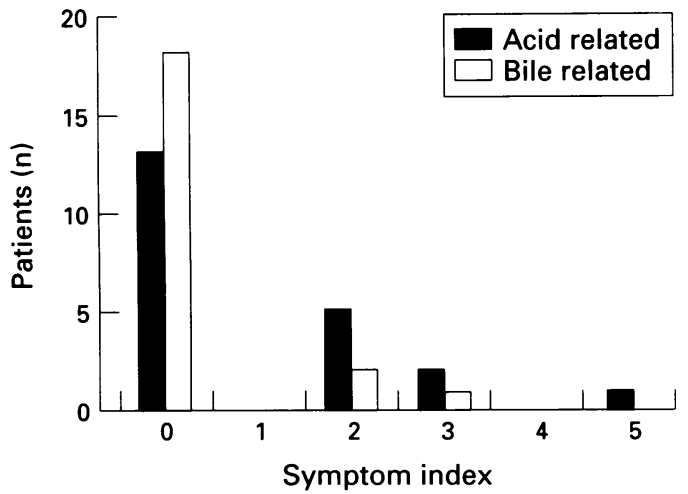

Figure 3: Symptom index scores for patients without significant gastro-oesophageal acid reflux (group 1). Note that for the majority of patients the score is very low whether related to acid or bile, indicating a poor correlation between symptom events and reflux episodes.

with 18 patients having an SI score of 0 . In other words, there was generally a very poor correlation between symptoms and reflux episodes of either acid or bile in group 1.

In group 2 on the other hand (Fig 4), 27 patients $(71 \%)$ had an acid related SI of $50 \%$ or greater (that is, a SI score of 3,4 or 5), whereas 32 patients $(84 \%)$ had a bile related SI of less than $50 \%$ (that is, a SI score of 0 , 1 or 2). In other words, it seems that in patients with significant acid reflux, symptoms are strongly related to acid reflux in most patients, but are poorly related to bile reflux in a greater majority.

\section{Discussion}

The relationship between acid reflux episodes and symptoms has long been established. Several methods of symptom analysis have been described in an effort to relate symptoms to episodes of acid reflux. The SI, defined as the percentage of reflux related symptom episodes, ${ }^{15}$ is the simplest form of symptom analysis. The drawback of this method is that it fails to take into account the total number of reflux episodes, with the possibility that a high SI may be the result of a random association between reflux and symptoms. The symptom sensitivity index, defined as the percentage of symptom associated reflux episodes, ${ }^{16}$ was introduced to try to overcome this drawback, but it fails to take into account the number of

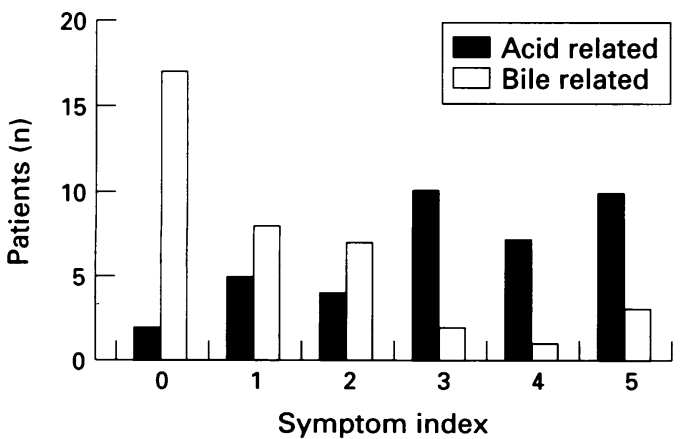

Figure 4: SI scores for patients with significant gastrooesophageal acid reflux (group 2). Note the generally low scores with regards to bile reflux, but the relatively high scores achieved by the majority of patients with regards to acid reflux. symptom episodes, thus limiting its value. Other techniques of symptom analysis have also been developed with the aim of improving the way in which symptoms and reflux can be related, but these have become increasingly complicated. ${ }^{17}{ }^{18}$ It was therefore felt that, for the purpose of merely comparing the relative contributions of acid and bile in the genesis of symptoms, the SI was an appropriate method of symptom analysis.

One of the difficulties in trying to relate bile reflux episodes to symptom events can be seen by looking at the segment of a 24 hour combined acid and bile reflux tracing in Figure 5. Acid reflux episodes are easily recognised definite, relatively fast, sharp downward deflections in $\mathrm{pH}$ from around $\mathrm{pH} 6$ or 7 to $\mathrm{pH} 4$ and below, often closely coinciding with a symptom event. Bile reflux episodes, however, are less well demarcated, and usually there is a smooth, gradual increase in bilirubin absorbance to above the threshold value of $0 \cdot 14$, which often lasts many minutes, rather than a sharp upward deflection. The other difficulty lies in the fact that an acid reflux episode from $\mathrm{pH} 7$ to $\mathrm{pH} 1$ represents, on a $\mathrm{pH}$ scale of $0-9$, a change of $66 \%$, whereas a bile reflux episode from, for instance, $0 \cdot 12$ to $0 \cdot 2$, on a scale of $0-1 \cdot 0$, represents a change of only $8 \%$. In other words bile reflux is said to occur in the presence of only a small increase in bilirubin absorbance. One of the reasons why bile reflux is not represented by a sharp deflection probably lies in the method of refluxate sampling. When measuring $\mathrm{pH}$, an antimony $\mathrm{pH}$ probe detects changes in the hydrogen ion concentration of the fluid directly surrounding the outer surface of the probe. Oesophageal clearance of the refluxate should therefore clear the outer surface of the $\mathrm{pH}$ probe with relative speed. This is compared with the bile probe, which relies on the oesophageal refluxate moving into the $2 \mathrm{~mm}$ gap within the bile probe tip, between the end of the optical fibres and the white Teflon probe head (Fig 6). Bearing in mind that, with associated mucus and particulate matter, oesophageal refluxate may be more viscous than pure water, it is probable that meniscal forces play a part in the movement of fluid into and out of the probe gap. Hence it is probable not only that a bile reflux episode may take longer to register than acid reflux episode, but also that oesophageal clearance of bile from the probe tip gap will take longer. All this would presumably result in a slower increase in bilirubin absorbance during an espisode of bile reflux in comparison with the sharp changes in $\mathrm{pH}$ observed during an acid reflux episode. This is the reason why, although the optimum time window for symptom analysis of 24 hour $\mathrm{pH}$ monitoring is considered to be the two minutes preceeding the symptom event, ${ }^{19}$ in this study we used a time window of two minutes either side of the symptom event. It should be noted, however, that despite this larger time window, symptom episodes were still much more frequently associated with acid reflux than with bile reflux.

Despite the fact that in group $129 \%$ and in group $262 \%$ of symptoms are related to a bile 

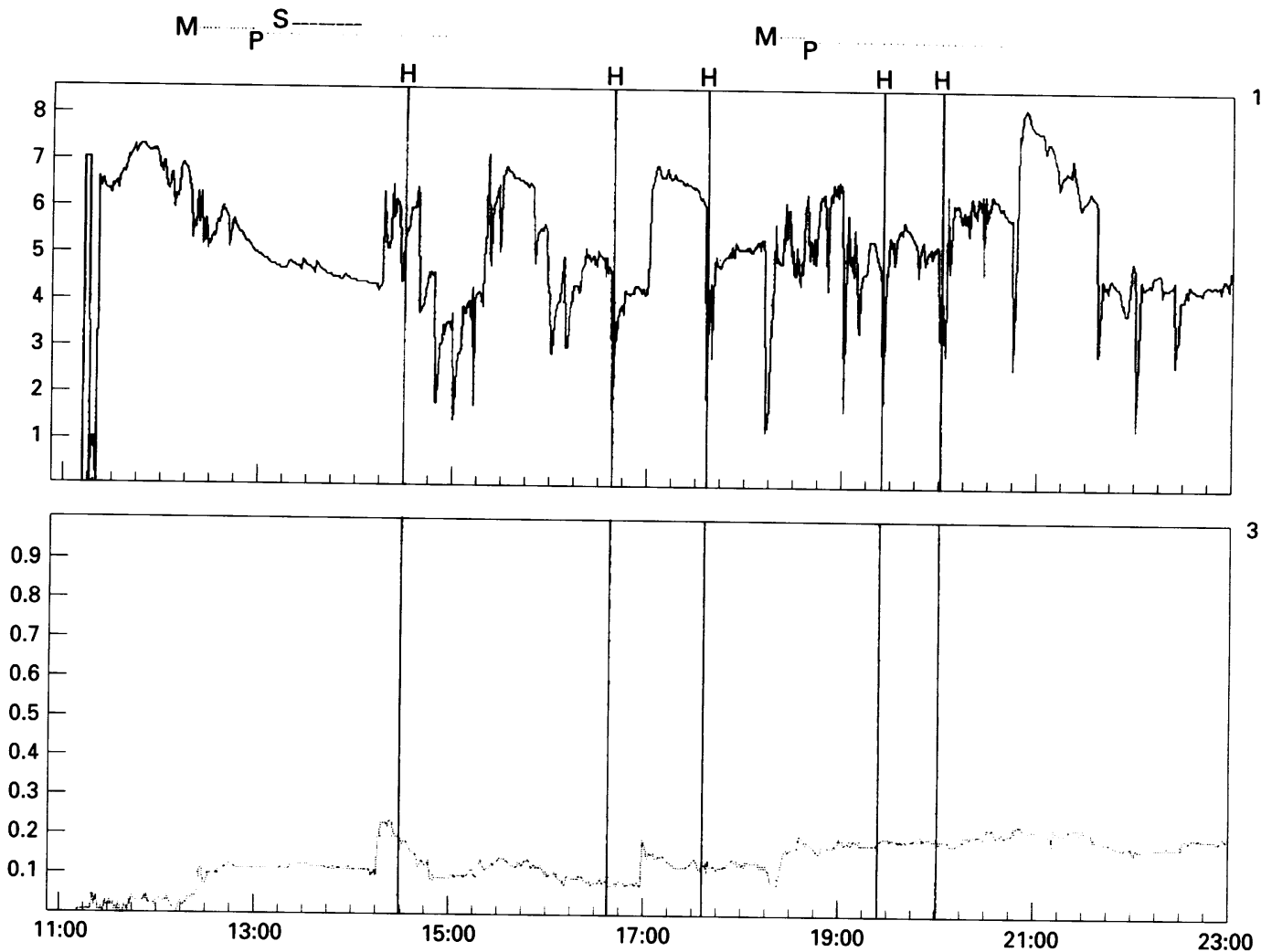

Figure 5: $A 12$ hour segment of a 24 hour combined $p H$ and bile monitoring (oesophageal pH above, bilirubin absorbance below) in a patient with gastro-oesophageal reflux. Note how acid reflux episodes are represented by sharp, easily identified falls in $p H$ whereas bile reflux, defined as a bilirubin absorbance $>0 \cdot 14$, occurs as slower, less discrete rises in absorbance.

or an acid reflux episode, or both, it should be recognised that the remainder are not, and an explanation for this should be sought. On many occasions, a reflux episode can be identified just outside the four minute time window, and it is possible that this is due to poor symptom recognition or inadequate registration of the event by the patient. Gastric, biliary or even cardiac disorders may well be responsible for some of the symptoms. It is interesting to see that similar proportions of

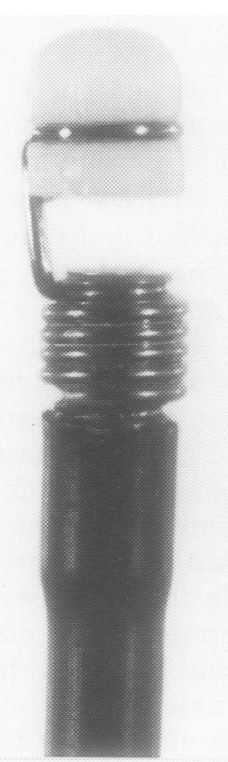

Figure 6: Photograph of an antimony $p H$ probe next to the larger Bilitec bile probe. Note the $2 \mathrm{~mm}$ gap between the white Teflon head and the end of the optical fibres in which the refluxate is sampled. each group had a positive Bernstein test. This may well reflect the comparatively poor sensitivity and specificity of this test in the detection of gastro-oesophageal reflux. ${ }^{20}$ In a study of patients with non-cardiac chest pain, Rokkas et $a l^{21}$ found that only $67 \%$ of patients with a positive Bernstein test had abnormal gastrooesophageal reflux, so it is possible that some of our patients in group 1 had a motility disorder that would have required ambulatory motility studies to detect.

There is a difference of opinion in the medical literature over the most appropriate protocol for the use of Bilitec 2000 in the measurement of oesophageal bile reflux. In particular, it has been disputed whether it is better to allow the patient to have an unrestricted diet, and then to exclude the meal and postprandial periods from the analysis, or whether to specify a diet that avoids interference with bilirubin absorbance and to include the whole 24 hour time period in the analysis. ${ }^{22}$ When 24 hour pH data are normally analysed, the whole 24 hours is included, and the results of this study of symptom analysis lend weight to the argument that this should be the case when analysing bile reflux data as well: $46 \%$ of all symptoms occurred in the meal and postprandial periods, and it is often these periods when the patients complain most bitterly of their symptoms. We believe, therefore, that patients should be instructed to have a diet that avoids interference with bilirubin absorbance so that the whole 24 hour period can be included in the analysis. As long as patients keep a good record of the foodstuffs they eat, any artefactual rise in bilirubin absorbance can be identified and excluded from the analysis. 
The good correlation between acid reflux and bile reflux reflects the results that have been published elsewhere using Bilitec 2000 . It is interesting to observe that despite this good correlation, the number of symptoms related to acid reflux is much greater than the number related to bile reflux. This is so not only in group 1 but also in group 2. One of the areas in which it was felt that bile reflux may play a part was in those patients who were symptomatic and yet had normal pH studies. In this series of patients with normal $\mathrm{pH}$ studies (group 1), only two of 21 patients had bile reflux, and yet in neither of these was there a strong SI with respect to bile reflux. It seems that not only is bile reflux in the absence of acid reflux a rare event in patients with an intact stomach, but that the bile itself is not often directly related to symptom events in these patients. A parallel can be drawn here with a study by Sears $e t a l^{23}$ who looked at acid and bile reflux in patients who had undergone previous partial gastrectomy. They observed that 10 of 13 patients had abnormal amounts of duodenogastro-oesophageal reflux and three of these 10 patients also had excessive gastrooesophageal acid reflux. It was only these three patients who had oesophagitis on endoscopy, and in these patients $97 \%$ of the symptoms were acid related. They concluded that although excessive amounts of duodenogastric reflux are common in partial gastrectomy patients, oesophagitis is found in those with concomitant acid reflux, and that most of the symptoms are acid rather than bile related.

In summary, although the physical characteristics of bile detection using the Bilitec 2000 system do not result in discrete reflux episodes such as are seen with acid reflux, bile itself does not seem to contribute to the symptomatology of oesophageal disease in the same way that acid does. It may be, of course, that the nature of oesophageal bile reflux is different from acid reflux, and the results of Bilitec are reflecting this. It should be emphasised that this study is concerned with symptoms, and does not attempt to draw any conclusions with regards to the role of bile in the pathogenesis of oesophageal mucosal damage. What seems to be certain, however, is that although there is a good correlation between the percentage times for oesophageal bile and acid reflux within people, bile and acid reflux does not always occur simultaneously, and the vast majority of symptom events are asociated with acid reflux episodes.
1 Mittal RK, Reuben A, Whitney JO, McCallum RW. Do bile acids reflux into the oesophagus? A study in normal subjects and patients with gastroesophageal reflux disease. Gastroenterology 1987; 92: 371-5.

2 Johnsson F, Joelsson B, Floren CH, Nilsson A. Bile salts in the oesophagus of patients with oesophagitis. Scand $\mathscr{f}$ Gastroenterol 1988; 23: 712-6.

3 Hamilton SR, Yardley JH. Regeneration of cardiac type mucosa and acquisition of Barrett's mucosa after oesophagogastrostomy. Gastroenterology 1977; 72: oesophagog.

4 Iftikhar SY, Ledingham S, Steele RJ, Evans DF, Lendrum K, Atkinson $\mathrm{M}$, et al. Bile reflux in columnar-lined Barrett's oesophagus. Ann R Coll Surg Engl 1993; 75: 411-6.

5 Miwa K, Hattori T, Miyazaki I. Duodenogastric reflux and foregut carcinogenesis. Cancer 1995; 75: 1426-32.

6 Houghton PW, Mortensen NJ, Thomas WE, Cooper MJ, Morgan AP, Davies P, et al. Intragastric bile acids and scintigraphy in the assessment of duodenograstric reflux. Br F Surg 1986; 73: 292-4.

7 Iftikhar SY, Ledingham S, Evans D, Yusuf SW, Steele RJC, Atkinson $\mathrm{M}$, et al. Alkaline gastro-oesophageal reflux: dual pH probe monitoring. Gut 1995; 37: 465-70.

8 Baldini F, Falciai R, Scheggi AM, Bechi P. A new fibre-optic sensor for bile reflux. In: Proceedings optical fiber sensors sensor for bile reflux. In: Proceedings optical fiber sensors
(OFS '88) (New Orleans, Louisiana). Norwood, Massachusetts: Artech House, 1988: 353-6.

9 Bechi P, Pucciani F, Baldini F, Cosi F, Falciai R, Mazzanti R, et al. Long-term ambulatory enterogastric reflux monitoring. Validation of a new fibreoptic technique. Dig Dis Sci 1993; 38: 1297-306.

10 Vaezi MF, Lacamera RG, Richter JE. Validation studies of Bilitec 2000: an ambulatory duodenogastric reflux monitoring system. Am f Physiol 1994; 267: G1050-7.

11 Caldwell MT, Byrne PJ, Brazil N, Crowley V, Attwood SE, Walsh TN, et al. An ambulatory bile reflux monitoring system: an in vitro appraisal. Physiol Meas 1994; 15: system:

12 Vaezi MF, Richter JE. Synergism of acid and duodenogastroesophageal reflux in complicated Barrett's esophagus. Surgery 1995; 117: 699-704.

13 Champion G, Richter JE, Vaezi MF, Singh S, Alexander R. Duodenogastroesophageal reflux: relationship to $\mathrm{pH}$ and importance in Barrett's oesophagus. Gastroenterology 1994; 107: 747-54.

14 Marshall REK, Anggiansah A, Owen WA, Owen WJ. Oesophageal acid and bile reflux correlate well: no relationship to alkaline pH. Gut 1996; 38: A25.

15 Wiener GJ, Richter JE, Copper JB, Wu WC, Castell DO. The symptom index: a clinically important parameter of ambulatory 24-hour esophageal pH monitoring. $A m \mathcal{F}$ ambulatory 24-hour esophageal

16 Breumelhof R, Smout AJ. The symptom sensitivity index: a valuable additional parameter in 24 hour oesophageal pH recording. Am $\mathcal{F}$ Gastroenterol 1991; 86: 160-4.

17 Johnston BT, McFarland RJ, Collins JSA. The binomia symptom index: correlating oesophageal symptoms with acid reflux. Gastroenterology 1992; 102: A92.

18 Weusten BL, Roelofs JM, Akkermans LM, Van BergeHenegouwen GP, Smout AJ. The symptom-association probability: an improved method for symptom analysis of 24-hour esophageal pH data. Gastroenterology 1994; 107: 1741-5.

19 Lam HG, Breumelhof R, Roelofs JM, Van Berge Henegouwen GP, Smout AJ. What is the optimal time
window in symptom analysis of 24-hour oesophageal window in symptom analysis of 24-hour oesoph
pressure and pH data. Dig Dis Sci 1994; 39: 402-9.

20 Richter JE, Hewson EG, Sinclair JW, Dalton CB. Acid perfusion test and 24-hour oesophageal $\mathrm{pH}$ monitoring with symptom index: a comparison of tests for oesophageal acid sensitivity. Dig Dis Sci 1991; 36: 565-71.

21 Rokkas T, Anggiansah A, McCullagh M, Owen WJ. Acid perfusion and edrophonium provocation tests in patients with chest pain of undetermined aetiology. Dig Dis $S_{c i}$ 1992; 37: 1212-6.

22 Marshall REK, Anggiansah A. Ambulatory oesophageal bile reflux monitoring in Barrett's oesophagus. Br $\mathcal{f}$ Surg reflux monitoring

23 Sears RJ, Champion GL, Richter JE. Characteristics of distal partial gastrectomy patients with oesophageal symptoms of duodenogastric reflux. Am $\mathcal{f}$ Gastroenterol 1995; 90: $211-5$. 